\title{
Genomic architecture and sexually dimorphic expression underlying immunity in the red mason bee, Osmia bicornis
}

\author{
Jannik S. Möllmann ${ }^{1^{*}}$ and Thomas J. Colgan ${ }^{1^{*}}$ \\ 1. Institute of Organismic and Molecular Evolution, Johannes Gutenberg University Mainz, \\ Hanns-Dieter-Hüsch-Weg 15, 55128 Mainz, Germany.

\section{*Corresponding authors:} \\ JSM: jmoellma@students.uni-mainz.de \\ TJC: tcolgan@uni-mainz.de
}

\section{Abstract}

Insect pollinators provide crucial ecosystem services yet face increasing environmental pressures.

The challenges posed by novel and reemerging pathogens on bee health means we need to improve our understanding of the immune system, an important barrier to infections and disease.

Despite its importance, for certain ecologically important species, such as solitary bees, our understanding of the genomic basis and molecular mechanisms underlying immune potential, and how intrinsic and extrinsic factors may influence immune gene expression is lacking. Here, to improve our understanding of the genomic architecture underlying immunity of a key solitary bee pollinator, we characterised putative immune genes of the red mason bee, Osmia bicornis. In addition, we used publicly available RNA-seq datasets to determine how sexes differ in immune gene expression and splicing but also how pesticide exposure may affect immune gene expression in females. Through comparative genomics, we reveal an evolutionary conserved set of more than 500 putative immune-related genes. We found genome-wide patterns of sex-biased gene expression, including immune genes involved in antiviral-defence. Interestingly, the expression of certain immune genes were also affected by exposure to common neonicotinoids, particularly genes related to haemocyte proliferation. Collectively, our study provides important insights into the gene repertoire, regulation and expression differences in the sexes of $O$. bicornis, as well as providing additional support for how neonicotinoids can affect immune gene expression, which may affect the capacity of solitary bees to respond to pathogenic threats.

Keywords: immunity, solitary bees, Osmia, sex differences, pesticide, gene expression 
Short title: Immune gene expression in the red mason bee.

\section{Introduction}

Insect pollinators provide key ecosystem services that are essential for the maintenance of agricultural crop yields, as well as natural biodiversity (Klein, Steffan-Dewenter and Tscharntke, 2003; Losey and Vaughan, 2006; Gallai et al., 2009). Pollination by insects, including social and solitary bees, is estimated to contribute $\$ 15.2$ billion to the US economy demonstrating the economic benefits provided by such services (Calderone, 2012). Despite the importance of such services, recent documented declines in bee populations have raised concerns over the continued provision of such services and related issues with food security (Vanengelsdorp and Meixner, 2010; European Red List of Bees, 2014; Goulson et al., 2015). Both abiotic and biotic factors have been highlighted as contributing factors to decline, including habitat loss and fragmentation, climate change, increased pesticide usage in modern agriculture, as well as pathogens and disease (Brown and Paxton, 2009; Goulson et al., 2015).

An important barrier to infection and establishment of disease is the invertebrate immune system (Rolff and Reynolds, 2009; Sadd and Schmid-Hempel, 2009). Despite lacking the adaptive immune system found in vertebrates, invertebrates have a dynamic innate immune system consisting of recognition molecules, signalling pathways and effector molecules, which coordinate the targeting and removal of potentially harmful entities (Hoffmann, 1995). In addition to the evolutionary importance of the immune system (Sadd and Schmid-Hempel, 2008; Viljakainen, 2015), understanding insect immunity has applied purposes, especially within the fields of biomedical, agricultural and conservation biology. Genomic studies on insects have provided novel insights into the genes and genomic architecture underlying the immune system (Christophides et al., 2002; Evans et al., 2006; Sackton et al., 2007; Waterhouse et al., 2007; Gerardo et al., 2010). Such studies have documented and helped understand the immune potential and capacity of a species through the identification of gene family expansions, contractions, as well as lineage-specific or novel genes that demonstrate immune function (Adams, 2000; Evans et al., 2006; Barribeau et al., 
2015). Indeed, comparative genomics allows for examining the types of selection pressures acting on such genes providing important insights into their evolutionary history. Given the enormous selection pressures placed upon hosts by pathogens (Combes, 2001), genes involved in the immune system are expected and have been observed to evolve under strong positive selection. Indeed, in comparative genomic studies of both vertebrates and invertebrates, including insects, immune genes are often identified with signatures of accelerated rates of evolution (Viljakainen et al., 2009; Roux et al., 2014; Shultz and Sackton, 2019).

Functional genomics, such as genome-wide transcriptional profiling ("RNA-seq"), provide highscale resolution of genome-wide changes in gene expression in response to immune or pathogen challenge but also intrinsic differences in expression between different life cycle stages or sexes (Fish, 2008; Klein and Flanagan, 2016). Indeed, sexually dimorphic gene expression has been identified across taxonomically diverse groups and may exist due to differences in life histories, hormonal abundance, biochemical reactions or sex-specific genomic architecture (Hill-Burns and Clark, 2009). Such differences can underlie differences in immune expression and function but also susceptibility to disease and related survival (Ingersoll, 2017). A striking example whereby sex-specific differences in genomic architecture may underlie differences in immune potential, activity and expression are members of the Hymenoptera, which include the bees, ants and wasps. Within this group, sexes differ in their ploidy with females developing from fertilised diploid eggs while males develop from unfertilised haploid eggs (Pamilo and Crozier, 1981). The haploid nature of males means that any alleles carried are automatically expressed and open to selection, which can result in the rapid removal of maladaptive deleterious alleles from the gene pool (Joseph and Kirkpatrick, 2004). The haploid nature of males has led to predictions that they are more susceptible to environmental challenges, such as pathogens (O'Donnell and Beshers, 2004), although empirical evidence to support this view has been conflicting (Baer et al., 2005; Calleri et al., 2006; Ruiz-González and Brown, 2006; Colgan et al., 2011; Retschnig et al., 2014).

Despite the fact that pathogen exposure and intrinsic differences can affect or influence immune gene expression, other factors can also have an influence, including nutritional status (Moret and 
Schmid-Hempel, 2000; DeGrandi-Hoffman and Chen, 2015), mating (Peng, Zipperlen and Kubli, 2005; Lawniczak et al., 2007; Barribeau and Schmid-Hempel, 2017), periods of senescence or dormancy (Nakamura et al., 2011; Kubrak et al., 2014; Colgan et al., 2019), as well as environmental factors, such as temperature (Xu and James, 2012; Chen, Nolte and Schlötterer, 2015). One environmental challenge that has received considerable attention of late is the influence of pesticide exposure on immune expression and function. Chemical pesticides, such as pyrethroids and neonicotinoids, interact with the insect nervous system resulting in paralysis and death (Matsuda et al., 2001). The efficacy of chemicals, such as neonicotinoids, combined with its lower toxicity to vertebrates, their systemic mode of action, as well as the lack of requirement for reapplication has resulted in their increased use in modern agricultural practices (Jeschke and Nauen, 2008). Despite their efficacy in killing agricultural pest species, the ubiquitous distribution of neonicotinoids across tissues means that non-target insects, including beneficial pollinators, may come in contact with such chemicals through food resources, such as nectar and pollen (Blacquière et al., 2012). The concentration of such chemicals can result in sublethal and indirect effects on the insect phenotype and has been identified to adversely affect the behaviour (Gill, Ramos-Rodriguez and Raine, 2012; Stanley, Smith and Raine, 2015; Arce et al., 2017, 2018; Siviter et al., 2018), neurobiology (Moffat et al., 2015, 2016) and gene expression of key ecological and commercial pollinators (Chaimanee et al., 2016; Beadle et al., 2019; Bebane et al., 2019; Colgan et al., 2019). In addition, such chemicals have been shown to affect immune expression and function (Di Prisco et al., 2013; Mason et al., 2013; Chmiel et al., 2019; Brandt et al., 2020) raising concerns that these effects may influence the ability of an exposed individual to respond to pathogenic threats (James and Xu, 2012). While studies have identified molecular mechanisms by which bees can metabolise certain neonicotinoids (Manjon et al., 2018; Beadle et al., 2019; Troczka et al., 2019), our understanding of changes in immune expression due to neonicotinoid exposure, especially for solitary bees, is limited.

The mason bees (Osmia species) are an important group of solitary bee pollinators but are generally understudied from an immunological perspective. One such species is the red mason 
bee Osmia bicornis (Order Hymenoptera; Family Megachilidae), a common pollinator found across central Europe, which has been increasingly incorporated into modern agricultural practices (Gruber et al., 2011). Despite its importance, it faces a number of environmental challenges that can influence the immune system, including pathogens and parasites (Seidelmann, 2006; Schoonvaere et al., 2018; Tian et al., 2018; Bramke et al., 2019), as well as pesticides (Brandt et al., 2020). Similarly, intrinsic differences between the sexes, which differ in morphology, physiology, behaviour and ploidy (Dmochowska-Ślęzak et al., 2015; Rogers, Frasnelli and Versace, 2016; Szentgyörgyi et al., 2017), may result in differences in immune expression and associated susceptibility to pathogenic threats. However, at present, our understanding of the immune gene repertoire and expression in $O$. bicornis is currently limited.

To improve our understanding on the immune potential of O. bicornis, we performed a comparative genomic analysis to identify the immune gene repertoire of the red mason bee as well as identify potential contractions and expansions of important gene families and determine whether the red mason bee is missing immune genes. Furthermore, to understand how genes underlying immunity may be expressed differently between the sexes, we investigated evidence of sex-biased gene expression and alternative splicing. Lastly, as pesticides can negatively affect different aspects of Osmia health, including developmental rate (Mokkapati, Bednarska and Laskowski, 2021), foraging behaviour (Boff et al., 2021; Straub et al., 2021), reproductive output (Sandrock et al., 2014; Woodcock et al., 2017; Ruddle et al., 2018), thermoregulation (Azpiazu et al., 2019), as well as impact immune function in red mason bees (Brandt et al., 2020), we examined whether neonicotinoid-exposed individuals differed in immune gene expression.

\section{Results}

\section{Putative expanded immune gene repertoire in the Hymenoptera}

To infer putative immune genes in 0 . bicornis, we independently examined the presence of homologues of previously characterised immune genes of the fruit fly, Drosophila melanogaster, and the closely related earth bumblebee, Bombus terrestris in the O. bicornis predicted proteome. 
We merged resulting $O$. bicornis homologues of known immune genes in $D$. melanogaster and $B$. terrestris, resulting in a total of 541 putative immune gene homologues, of which 99 were only found among the set of $B$. terrestris immune genes, 387 were only found among the set of $D$. melanogaster immune genes and 55 were found among both sets (Figure 1A, Supplemental File S1). We also ran functional enrichment analyses and found enrichment in immune terms for the putative immune genes derived uniquely via homology to $D$. melanogaster and for the putative immune genes found among both sets while for the putative immune genes derived uniquely via homology to $B$. terrestris we found enrichment in terms only indirectly linked to the immune system such as "response to stress" and "response to toxic substance" (Supplemental File S2).

Given that previous genomic studies on immune gene repertoires in hymenopterans have described lower gene counts, and since $O$. bicornis is more evolutionarily distantly related to $D$. melanogaster than B. terrestris, we further examined immune genes identified only through homology to $D$. melanogaster to determine confidence in homology. For this, we examined the sequence identity of homologous immune proteins as calculated by OrthoFinder between $O$. bicornis and $D$. melanogaster revealing higher percentage sequence identity for putative immune proteins found only in the set of $D$. melanogaster immune proteins compared to immune proteins found uniquely in the set of canonical B.terrestris immune proteins or immune proteins found in both sets (Figure 1D). As metrics of sequence identity can be influenced by protein length, we compared predicted protein lengths between homologous pairs, revealing a strong positive correlation (Pearson's Product Moment Correlation Coefficient, $\mathrm{R}=0.94, p<2.2 \mathrm{e}-16$, Figure 1B-C). Lastly, we examined if homologous sequences shared the same type and number of functional domains, which would potentially suggest conserved function. We found identical domain annotations for a high percentage $(71.01 \%, \mathrm{n}=722)$ of all homologous pairs between 0 . bicornis and $D$. melanogaster with on average $90.05 \%$ of the domains shared between pairs.

Our analysis also revealed immune genes potentially missing in $O$. bicornis. We did not identify homologues for $232 D$. melanogaster immune genes as well as one canonical $B$. terrestris immune gene, the antimicrobial peptide abaecin, in O. bicornis or its sister taxa, O. lignaria (Supplemental 
File S1). Inversely, as Osmia may contain lineage specific genes, including genes with potential immune functions, we identified genes ( $n=78$, split across 48 orthogroups; Supplemental File S1) shared between $\mathrm{O}$. bicornis and $\mathrm{O}$. lignaria that lacked homologues in the predicted proteomes of all other 19 insect species we used to infer homology relations (for species list see Experimental Procedures). Among the Osmia-specific genes that were annotated with at least one domain $(n=24)$, we find 16 genes annotated with ribonuclease-domains (IPR036397, IPR012337) as well as one gene (LOC114879997) annotated with a rhabdovirus nucleoprotein domain (IPR004902).

To understand variation in the immune gene complement of the red mason bee, we compared the number of putative $O$. bicornis immune genes in conserved gene families and pathways to the number of homologues in three closely-related hymenopteran species $(O$. lignaria, $B$. terrestris and A. mellifera) and one more distantly-related fly species (D. melanogaster). We found similar numbers of genes in the four hymenopterans for most signaling pathways or non-pathway gene families, with slightly lower gene numbers in the two Osmia species compared to the other two hymenopterans for the Immune deficiency (ImD) and Toll pathways (Figure 2A) and higher numbers for inhibitors of apoptosis in $B$. terrestris $(n=13)$ compared to the other hymenopterans ( $\mathrm{n}=4$ to 5 , Figure $2 \mathrm{~B}$ ). When comparing the hymenopterans to $D$. melanogaster, the latter has higher gene counts for six out of 14 signaling pathways and 13 out of 22 non-pathway gene families but similar numbers for all other pathways and gene families. In addition, we checked the number of homologues in $O$. bicornis for $D$. melanogaster immune genes on a gene family level and found a high average conservation of $84.86 \%$ but a large difference for antimicrobial peptides with only one O. bicornis homologue compared to 22 D. melanogaster genes.

\section{Sex-biased differential expression of immune genes}

To provide functional information on the expression of putative immune genes in 0 . bicornis, we compared whole-bodied transcriptomes of male $(n=3)$ and female adults $(n=4)$. We identified 4,128 genes $(34.99 \%$ of total gene count, $n=11,799)$ as significantly differentially expressed (Likelihoodratio test, $\mathrm{BH}$-adjusted $p<0.05$ ) between the sexes, of which, 2,087 and 2,041 had female- and male-biased expression, respectively (Supplemental File S3). Among the differentially expressed 
genes, we found a significant enrichment or depletion (Kolmogorov-Smirnov test, $p<0.05$ ) of 125 biological process-associated GO terms, 47 cellular component $\mathrm{GO}$ terms and 29 molecular function GO terms with "cytoplasmic translation", "cytosolic large ribosomal subunit" and "structural constituent of ribosome" as the most enriched terms for each of the three ontologies, respectively (Supplemental File S2). We quantified expression of 520 putative immune genes $(96.11 \%$ of total immune genes, $n=541)$ in both sexes, of which 222 were differentially expressed $(42.69 \%$ of total DEGs) which was significantly more than expected by chance (Fisher's Exact test, $p=0.017$ ). These differentially expressed genes were nearly equally shared between the sexes with slightly more genes $(n=118)$ showing male-biased rather than female-biased expression $(n=104)$, a pattern which did not significantly differ from expectation (Fisher's Exact test, $p=0.554$, Supplemental File S1). Among the differentially expressed immune genes, we found enrichment or depletion for 28 biological process $\mathrm{GO}$ terms, five cellular component GO terms and five molecular function GO terms with "RNA localization", "intracellular non-membrane-bounded organelle" and "RNA binding" as top enriched terms of each of the ontologies, respectively (Figure 3B, Supplemental File S2). We also analysed the Osmia-specific genes for signatures of differential expression and found 15 genes that significantly differed in their expression between the sexes (Supplemental File S1).

\section{Sex-biased alternative splicing of immune genes}

We also performed alternative splicing analysis and identified 1,019 genes $(8.64 \%$ of total gene count, $n=11,799$ ) significantly differentially spliced (Likelihood-ratio test, FDR $<0.05$ ) between the sexes (Supplemental File S4). These sex-biased genes were significantly enriched or depleted (Kolmogorov-Smirnov test, $p<0.05$ ) for 107 biological process $\mathrm{GO}$ terms, 24 cellular component GO terms and 18 molecular function GO terms with "sarcomere organisation", "Z disc" and "actin binding" as the top enriched terms for each ontology, respectively, as well as "immune system process", significantly enriched among the biological process GO terms (Supplemental File S2). We identified 71 putative immune genes as differentially spliced $(13.12 \%)$ between the sexes, which is significantly more than expected (Fisher's exact test; $p=0.002$ ). In addition, we found differences between the sexes in the frequency of different splicing events with retained intron 
events significantly more common in females than in males (Fisher's exact test, BH-adjusted $p=0.036$ ). For other splicing events, we found a borderline significant male-bias for alternative 3' splice sites (Fisher's exact test, $\mathrm{BH}$-adjusted $p=0.051$ ) while we found no significant differences (BH-adjusted $p>0.05)$ for skipped exons, alternative 5' splice sites and mutually exclusive exons.

\section{Immune gene expression changes in response to pesticide exposure}

For the neonicotinoid exposure analysis we identified 617 genes, including 42 putative immune genes, significantly differentially expressed (Wald-test, $\mathrm{BH}$-adjusted $p<0.05$ ) in the group of thiacloprid-exposed females compared to the untreated control group (Figure 4C, Supplemental File S3), with a significantly unequal partitioning of 436 genes up-regulated and 181 genes downregulated in the thiacloprid-exposed group (Binomial-test, $p=9.41 \mathrm{e}-22$ ). Comparing imidaclopridexposed females with untreated females, we found 127 genes significantly differentially expressed (Wald-test, $\mathrm{BH}$-adjusted $p<0.05$ ), including seven immune genes (Figure 4A). We also found a significantly unequal partitioning of 89 genes up-regulated and 38 genes down-regulated in the imidacloprid-exposed group (Binomial-test, $p=6.97 \mathrm{e}-5$ ). We found more differentially expressed immune genes than expected by chance in the thiacloprid-exposed group ( $n=42$ immune genes; Fisher's exact test, $p=0.014$ ) but not in the imidacloprid-exposed group ( $\mathrm{n}=$ seven immune genes; Fisher's exact test, $p=0.515$ ). Five putative immune genes (LOC114878095, LOC114878683, LOC114881181, LOC114874985, LOC114872156) had increased transcript expression both in response to thiacloprid and imidacloprid with no significant difference between the log2 fold change values for these five genes between the two pesticide treatment groups (Welch's t-test, $p=0.76$, Supplemental Files S1, S3). In terms of functional enrichment of significantly differentially expressed immune genes, we found 45 biological process-associated $\mathrm{GO}$ terms enriched or depleted for the thiacloprid-exposed group, as well as five cellular component and eight molecular function terms with "regulation of hemocyte proliferation", "integral component of plasma membrane" and "signaling receptor activity" as top enriched terms for each ontology, respectively (Figure 4D). For the imidacloprid-exposed group, we found 30 biological process $G O$ terms enriched or depleted among the differentially expressed immune genes as well as four cellular 
component terms and seven molecular function terms with "transmembrane receptor protein tyrosine kinase signaling pathway", "integral component of plasma membrane" and "signaling receptor activity" as top terms of each of the ontologies, respectively (Figure 4B, Supplemental File S2). Among the Osmia-specific genes we identified three genes differentially expressed between the thiacloprid-exposed group and control group but no differentially expressed genes between the imidacloprid-exposed group and the control group (Supplemental File S1).

For alternative splicing, we found 142 differentially spliced genes (Likelihood-ratio test, FDR<0.05) for the thiacloprid-exposed group, including six putative immune genes, while 74 genes were differentially spliced for the imidacloprid-exposed group, including four putative immune genes. One immune gene (LOC114880106) was differentially spliced in response to both pesticides (Supplemental File S4).

\section{Discussion}

The insect immune system represents an important barrier against infections and disease and thus provides a physiological function vital to an individual's success. While our understanding of the genomic and molecular bases of immunity in the Hymenoptera has largely been informed by studies on social bees, for other species, especially solitary bees, such information is limited. Here, we performed a comparative genomic analysis to characterise genes with potential immune functions in the genome of the red mason bee, O. bicornis. Using a homology-based approach, we identify an immune gene repertoire enlarged beyond the canonical immune genes previously described in other hymenopteran genomes. We find extensive differences in immune gene expression between the sexes, both in terms of expression amplitude and splicing, highlighting intrinsic regulatory differences in the molecular basis of immunity between males and females. Lastly, we find immune-related genes differentially expressed in response to neonicotinoid exposure with greater expression differences in bees exposed to thiacloprid than those exposed to imidacloprid demonstrating differences in how the molecular phenotype responds to different neonicotinoid subclasses and how each may influence immune expression. 
The insect immune system consists of an innate immune response with the ability to detect and remove a diverse range of pathogenic entities (Beckage, 2008). In addition to behavioural, physical and chemical defences, it is an important barrier to infection and disease development. The earliest genomic studies on the Hymenoptera documented a reduction in canonical immune genes in comparison to other insect orders, most notably in comparison to members of the Diptera (Evans et al., 2006; Bonasio et al., 2010; Werren et al., 2010; Barribeau et al., 2015). Reasons for this reduction ranged from the technical (e.g., fragmented genome assembly, missing or truncated gene models) to the biological level (e.g., novel immune genes and pathways ((Albert et al., 2011; Dong et al., 2020) or relaxed selection acting on canonical immune genes due to social immunity (Harpur and Zayed, 2013)). Here we performed one of the first investigations of the immune gene repertoire of a solitary bee species.

Our initial approach for the detection of putative immune genes was based on homology with genes annotated with roles in immune system function based on Gene Ontology in the model organism, $D$. melanogaster, where many such genes have been experimentally validated with roles in immune function. As many immune genes have been previously shown to evolve under strong episodic positive selection (Jiggins and Kim, 2007; Viljakainen, 2015; Shultz and Sackton, 2019), which can result in divergence beyond detection through homology searches or the appearance of novel lineage-specific immune genes, we also investigated homologues in $O$. bicornis of canonical immune genes from the earth bumblebee, B. terrestris, a closely related social insect with an annual life-cycle. As the majority of canonical immune genes identified in $B$. terrestris were generated based on homology searches with other insect genomes, including $D$. melanogaster (Barribeau et al., 2015), we would have expected canonical immune genes in both species to be annotated with immune process GO terms and therefore, we would have expected to see a high overlap in immune gene sets. Surprisingly, we found a weak overlap between Osmia homologues identified using both approaches, with only a third of $B$. terrestris canonical immune genes identified also through homology to $D$. melanogaster immune genes and thus annotated with immune system GO terms. The other two thirds were annotated with GO terms associated 
only more indirectly with immunity, such as "response to toxic substance", "RNA interference" and "autophagy". The lack of annotation of immune GO terms for two thirds of the described canonical immune genes in $B$. terrestris may result in the underreporting of immunological changes for genomic studies reliant on GO term based analyses.

To provide better support for conserved function for putative immune homologues in Osmia, we further assessed $O$. bicornis immune homologues identified through the $D$. melanogaster comparison, which lacked a described homologue in $B$. terrestris canonical immune genes. If such homologues were spurious or low quality matches, we predicted such protein homologues may have lower percentage sequence identity, greater differences in sequence length, as well as variation or lack of structural features, such as abundance and diversity of functional domains compared to canonical immune genes. However, for the majority of homologous pairs, we found the same or greater percentage sequence identity as canonical immune genes, as well as the presence and conservation of functional domains, suggesting that potential immune functions may be conserved and perform similar roles in bees. Identified through homology with $D$. melanogaster immune genes only, we found homologs of many immune relevant genes like defensins, hemocytin and sickie known to be important to the immune system across different insect species (Hoffmann and Hetru, 1992; De Gregorio et al., 2002; Lavine and Strand, 2002; Arai et al., 2013; $\mathrm{Ni}$ et al., 2020). Through homology with B. terrestris immune genes only, however, we found homologues of immune genes known to be involved in the insect immune system, like mucins, galectin-like proteins and superoxide-dismutases (Pace and Baum, 2002; Korayem et al., 2004; Colinet et al., 2011; Rao et al., 2016), demonstrating the importance of using more than one species to infer undescribed gene sets via homology. While future experimental studies on their function will elucidate potential roles in immunity, if any, for these additional candidate genes, their high number could also speak to the ever-increasing completeness of functional annotation in insect model organisms, like $D$. melanogaster.

Among the 541 putative immune genes of 0 . bicornis, we did not find any major patterns of immune gene family expansions or contractions in comparison with that of $B$. terrestris and $A$. 
mellifera, suggesting that there were no large-scale recent duplications or losses of genes that could be imperative to the functioning of the immune system of bees. However, we did find slight reductions in the number of genes involved in two major immune signaling pathways, Imd and Toll, when we compared $O$. bicornis and $O$. lignaria to $A$. mellifera and B. terrestris. Imd and Toll are both involved in the induction of antimicrobial peptides (AMPs) (De Gregorio et al., 2002), thus, fundamental for insect survival in response to pathogen challenge. Related to this, the biggest difference in immune gene families between $O$. bicornis and $D$. melanogaster was for gene copies of AMPs. This can be expected as Drosophila have evolved a number of AMP gene families, such as the cecropins, diptericins and attacins (Imler and Bulet, 2005), which have not been identified in other hymenopteran genomes (Evans et al., 2006; Barribeau et al., 2015). Consequently, we did identify the presence of defensin, an evolutionary conserved AMP that possesses antibacterial properties (Hoffmann and Hetru, 1992), as well as the hymenopteran-specific AMP, hymenoptaecin (Casteels et al., 1993). However, we did not detect a copy of abaecin, a bacterialinducible AMP described in honeybees (Casteels et al., 1990) and bumblebees (Rees, Moniatte and Bulet, 1997). The conserved lack of abaecin, as well as components of the Imd and Toll signaling pathways, in the genome assemblies of two Osmia species, which were sequenced and assembled independently, suggests that such missing genes may be true biological signals rather than technical artefacts. At present the evolutionary consequences of such potential losses, if any, are unknown, but it suggests at least that differences in the molecular structure of the immune system do indeed exist for these two solitary bee species compared to these other social bee species.

For species that sexually reproduce, the genome codes for distinct sexes that can differ dramatically in behaviour, morphology and physiology (Parsch and Ellegren, 2013), including immunity (Klein and Flanagan, 2016). As immunity can be both energetically costly to maintain and activate (Rolff and Siva-Jothy, 2003), it can result in metabolic trade-offs with other processes, such as reproduction (Schwenke, Lazzaro and Wolfner, 2016). Sexes can also differ in immune investment, which may be reflected in differences in gene expression. Here we found 222 putative 
immune genes to be differentially expressed between the sexes with the strongest functional enrichment for terms related to localization and breakdown of RNA and general macromolecules. This suggests that the sexes may differ in important housekeeping roles, such as RNA and protein turnover, but also how they respond to challenging macromolecules that need to be localized and decomposed, such as RNA-viruses and toxins. The case for differences in antiviral defence is further highlighted by genes, such as endonuclease Dcr-1 or defensin, which have roles in virus recognition and degradation (Galiana-Arnoux et al., 2006; Brutscher, Daughenbaugh and Flenniken, 2015), being differentially expressed between the sexes. We found more putative immune genes than expected by chance to be differentially expressed or differentially spliced between the sexes suggesting that the molecular differences between the sexes are particularly pronounced when it comes to immune system processes. We also looked at the expression differences between sexes in general and found the most striking functional enrichment of differentially expressed genes to be related to translational and cell division processes, underlining the generality of molecular differences between the sexes while the most striking enrichment of alternatively spliced genes is mostly related to muscle activity and regulation of muscle excitation which might reflect the different life strategies of male and female mason bees where male bees emerge prior to female bees or thermoregulatory differences.

Pesticides, including neonicotinoids, act as agonists of the nicotine acetylcholine receptors, resulting in disruption of the neuronal cholinergic signal transduction and excitation of neuronal triggers culminating in paralysis and death (Matsuda et al., 2001). The efficacy of the mode of action of neonicotinoids has led to their increased popularity in modern agriculture practices yet recent studies have highlighted the negative impact sublethal and lethal doses can have on pollinator health ('Neonicotinoids, bee disorders and the sustainability of pollinator services', 2013), including immune function. Neonicotinoids, such as clothianidin and imidacloprid, have been identified in exposed honeybees to negatively modulate the NF-kappaB signaling pathway and affect the ability to mount effective antiviral defences (Di Prisco et al., 2013). Other studies on neonicotinoids have provided additional evidence of the indirect or direct effects of these 
neurotoxins on immune function or expression (Brandt et al., 2017, 2020). Here we found changes in gene expression in response to two classes of neonicotinoids with thiacloprid exposure affecting the expression of more genes, including immune genes, than imidacloprid. This is in line with other studies that have looked at thiacloprid exposed red mason bees and observed impairment in immunity (Brandt et al., 2020) or larval development (Claus et al., 2021). For both neonicotinoids, we find significantly more genes up-regulated in response to pesticide exposure than expected, suggesting that overall the exposure to pesticides results in an active response of heightened gene expression as opposed to a mere passive shift in gene expression. Focusing on immune system processes, we find more immune genes differentially expressed than expected only in thiaclopridtreated individuals, suggesting that thiacloprid elicits a stronger immune response than imidacloprid. Interestingly, of the seven differentially expressed genes with elevated expression in imidacloprid-exposed bees, five genes were also significantly up-regulated in the thiaclopridexposed bees which could possibly point to a common set of immune genes that are up-regulated in response to neonicotinoid exposure. In terms of functional enrichment of the differentially expressed immune genes, both pesticide-treated groups share similar terms, related to signaling of transmembrane receptors, suggesting that the immune genes play a role in signaling in response to pesticide exposure. In addition, in the thiacloprid-treated group the term "regulation of hemocyte proliferation" is the most significantly enriched term. Haemocytes fulfill an important role in the ingestion and break-down of foreign cells and substances in the insect immune system (Lavine and Strand, 2002). Brandt et al. found a reduction in haemocyte density in red mason bees after exposure to thiacloprid, which is in line with our finding of haemocyte proliferation being differentially regulated between thiacloprid treated and untreated individuals, suggesting that the effect of pesticide exposure on haemocyte function may extend down to the molecular level.

\section{Conclusions}

The red mason bee, $\mathrm{O}$. bicornis, is a commercially and ecologically relevant solitary bee species, whose immune system has not been well-studied yet, albeit being integral to its future chances of survival when faced with increasing environmental challenges. Here, we utilised a comparative 
genomic approach to propose a set of genes as part of the immune gene repertoire of O. bicornis, and used RNA-seq data to show that the expression and regulation of these putative immune genes differs markedly between sexes and responds with heightened expression to treatment with two neonicotinoid pesticides. Additionally, our findings provide support for the application of a combined approach to inference of gene families, using homology information of more than one species of reference. Future studies on $\mathrm{O}$. bicornis immunity will benefit from tissue-specific profiling, as well as tracking gene expression changes in response to different immune challenges. Similarly the application of population genomics will provide important insights into the recent selection pressures acting on immune genes of mason bees. Collectively, our study provides novel insights into the immune system of an important, yet still understudied solitary bee species and identifies a candidate repertoire of immune genes for future research on the immune system of the red mason bee.

\section{Experimental Procedures}

\section{Identification of putative immune genes in the red mason bee}

To infer homologues for O.bicornis genes that are in other insect species, we ran OrthoFinder [v.2.5.2](Emms and Kelly, 2019) with proteomes of 21 species, comprising 11 bee species from three families (Family Megachilidae: Osmia bicornis, Osmia lignaria, and Megachile rotundata; Family Apidae: Apis mellifera, Ceratina calcarata, Eufriesea mexicana, Habropoda laboriosa and Bombus terrestris; and Family Halicitidae: Megalopta genalis, Nomia melanderi and Dufourea novaeangliae), as well as 10 non-bee insects (Drosophila melanogaster, Aedes aegypti, Anopheles gambiae, Bombyx mori, Tribolium castaneum, Acyrthosiphon pisum, Nasonia vitripennis, Solenopsis invicta, Polistes dominula and Vespa mandarinia). All proteomes were obtained from the National Center for Biotechnology Information (NCBI) Reference Sequence (RefSeq) database. We ran OrthoFinder using the default parameters with the inferred species trees forming a consensus with the known phylogeny. Given that model organisms, such as $D$. melanogaster, contain the most comprehensive functional annotation of genes with immune 
function or potential, we examined the O. bicornis predicted proteome for putative homologues of D. melanogaster immune genes. To obtain D. melanogaster immune-responsive genes, we queried the FlyBase on the 9th of July, 2021 for any gene associated with the GO term "immune system process", the highest order Gene Ontology term associated with the immune system, and inferred the O. bicornis homologues via the homologues table generated earlier. As an additional approach, to identify immune genes that may be lineage-specific within the Hymenoptera or too divergent between $O$. bicornis and $D$. melanogaster given their evolutionary distance, we examined the presence of $\mathrm{O}$. bicornis homologues from comparison with canonical immune genes characterised in the earth bumblebee, Bombus terrestris. The canonical B. terrestris immune genes were directly obtained from the most recent earth bumblebee genome papers (Barribeau et al., 2015; Sadd et al., 2015). An additional reason for the inclusion of this social bee species is that it has a curated homologue list with $D$. melanogaster, available through the Ensembl Metazoa database, which provided the ability to compare the orthogroups and homologous pairs generated by OrthoFinder with orthogroups and pairs independently generated by Ensembl, which incorporates an additional information on synteny and gene order conservation for the identification of putative homologues between two species. This approach identified a high overlap (86.85\% of Ensembl pairs correctly identified by OrthoFinder) between the pairs generated by both analyses providing additional confidence in the orthogroups generated by OrthoFinder. Homologous genes from $D$. melanogaster and B. terrestris were obtained by first translating the O.bicornis gene-IDs to protein-IDs via the annotation column in the RefSeq gene feature file (GFF) and then using the homology information from OrthoFinder to translate O. bicornis protein-IDs to D. melanogaster and B. terrestris protein-IDs respectively and further translating the protein-IDs to the species-specific gene-IDs, yielding a many-to-many homologue table of O. bicornis gene-ID's to D. melanogaster and B. terrestris gene-ID's, respectively. To infer for each gene family how many homologous genes exist for the set of putative $O$. bicornis immune genes in $A$. mellifera, $B$. terrestris, D.melanogaster and O. lignaria, we derived annotation of $D$. melanogaster genes with gene families from Flybase on the 02nd of June, 2021 and annotated the immune gene homologues in 
each species with the according gene family description. We then summarised this data by counting the number of genes in each species and for each gene family.

For $O$. bicornis genes that shared homology with $D$. melanogaster immune-responsive genes but did not overlap with known canonical immune genes in B. terrestris , we further examined homology based on the following criteria: 1) similarity of predicted protein length between homologous pairs; 2) high percentage of protein sequence identity between homologous pairs as inferred via Diamond searches performed by OrthoFinder; and 3) the number of shared functional protein domains between homologous as inferred via InterProScan [v5.52-86.0](Jones et al., 2014). The prediction here is that if two homologous proteins shared similar protein length, high sequence identity and the same or similar number and types of functional protein domains, potential functional immune roles may be conserved.

In addition to identification of immune-related genes, we also inferred canonical immune genes from B. terrestris or $D$. melanogaster missing in $O$. bicornis and $O$. lignaria. For this, we parsed the output of OrthoFinder for orthogroups that carried immune-associated genes in D. melanogaster or B. terrestris but not in both O. bicornis and O.lignaria. Similarly, we inferred Osmia-specific genes by parsing orthogroups containing only $\mathrm{O}$. bicornis and $\mathrm{O}$. lignaria homologues, which were also absent in the other 19 species.

\section{Quality assessment, transcript abundance estimation and differential expression} analysis of immune genes between sexes and pesticide-treated groups

To examine the functional expression of putative immune genes of $O$. bicornis, we obtained publicly available paired-end RNA-seq libraries for two analyses: a) sex-biased analysis containing males ( $n=3)$ and females $(n=4)$; and b) pooled libraries of unexposed ("control"; $n=4)$ females or those exposed to thiacloprid $(n=4)$ or imidacloprid $(n=4)$. All datasets were obtained from the NCBI (National Center for Biotechnology Information) Short Read Archive (SRA) database (BioProject: PRJNA285788; (Beadle et al., 2019), Supplemental File S5). We performed data quality assessment based on per-sample quality evaluations using FastQC [v.0.11.9](Andrews, 2010) 
calculation of the proportions of reads mapping to the predicted transcriptome of the RefSeq $O$. bicornis reference genome assembly [Obicornis_v3; GCF_004153925.1] using Kallisto [v.0.46.1] (Bray et al., 2016). We then combined and visualized the results for both tools and across all samples with MultiQC [v.1.7](Ewels et al., 2016). Based on the results of the quality assessment we removed adapter sequences and filtered by quality (phred quality score $>=15$ ) and length (minimum length $>=50 \mathrm{bp}$ ) using fastp [v.0.20.1](Chen et al., 2018). For each sample, we then aligned the trimmed and filtered reads against the most recent chromosome-level genome assembly [iOsmBic2.1; GCA_907164935.1] using STAR [v.2.7.8a](Dobin et al., 2013). As the chromosome-level assembly currently lacks annotations, we first transferred gene coordinates from the annotated reference assembly [Obicornis_V3; GCF_004153925.1] to the new assembly using Liftoff [v.1.6.1](Shumate and Salzberg, 2020). STAR was ran in two-pass-mode using the inferred splice junctions from the first run to improve the alignment of the second run and with parameter --quantMode GeneCounts used to generate gene level abundances of aligned reads (Supplemental File S6). The mean alignment rate across all samples was $94.09 \%$. For the sexbiased analysis, we used DESeq2 [v.1.30.1](Love, Huber and Anders, 2014) to correct for library size and infer all differentially expressed genes ("DEG", FDR < 0.05) between sexes with a likelihood-ratio-test (LRT; full model: sex; reduced model: intercept). For the pesticide-based analysis, we implemented pairwise Wald tests to determine log2 fold changes between each pesticide treatment and control, as well as quantify all differences in gene expression between pesticide treatments. For each analysis, we then parsed DEGs for putative immune genes. To determine if we identified more or less immune genes than would be expected, we performed Fisher's exact tests for each analysis.

As a complementary approach to STAR, we also implemented a pseudoalignment-based differential gene expression analysis using Kallisto [v.0.46.1] as described above in the quality assessment section (mean mapping rate across samples $87.93 \%$ ). Similar to the STAR-based analysis, we implemented the same statistical tests using DESeq2 for both the sex-biased and pesticide-based analysis, respectively. Out of the genes predicted to be differentially expressed 
using STAR, 3376 genes $(81.78 \%, n=4128)$ were also reported to be differentially expressed using Kallisto and inversely, $92.01 \%(n=3669)$ of all genes predicted to be differentially expressed using Kallisto were also predicted to be differentially expressed using STAR.

\section{Splicing of immune genes between sexes and pesticide-treated groups}

To determine differentially spliced genes between the sexes, as well as in response to pesticide exposure, we ran rMATS turbo [v.4.1.1](Shen et al., 2014) using the STAR generated alignment files. Similar to our differential expression analyses, we performed two independent analyses: first, we compared males and females to identify significant sex-biased differences in splicing; second, we performed a pesticide-based analysis, comparing thiacloprid- and imidacloprid-exposed females against the control group, respectively. A specific feature of rMATS is that it outputs eventlevel instead of gene-level results, distinguishing between different event types such as exonskipping, intron-retainment and 3'- or 5'-splicing and allowing multiple splicing events to be recognized per gene. We used this event-based information to investigate whether there were incidences of alternative splicing where a specific splicing event appears preferentially in one of the experimental groups over the other. To do this we compared the number of times a differential splicing event had higher inclusion levels in one group over the other against the total number of incidences of differential splicing for that event using a Binomial-test with the null hypothesis being that we would see higher inclusion levels equally often for both groups. We then concatenated and summarised the event-level results by grouping the events by gene-ID and summarising down to the lowest $p$ value per gene, yielding a gene-level output table.. We then used this information to rank genes by $p$ value and investigate functional enrichment of Gene Ontology (GO) terms in alternatively spliced genes (below).

\section{Gene Ontology term enrichment analysis}

As most genes in the $\mathrm{O}$. bicornis genome lack functional information, we assigned $\mathrm{GO}$ terms to genes from homologues found in $D$. melanogaster, which were obtained from Ensembl Metazoa via biomaRt [v.2.46.3](Durinck et al., 2009; Kinsella et al., 2011). We then ran functional enrichment analyses using topGO [v.2.42.0; (Alexa and Rahnenführer, 2009)] on the sets of 
immune genes derived via homology to A) D. melanogaster uniquely, B) $B$. terrestris uniquely and C) to both species. To do so we implemented the "classic" algorithm in combination with a Fisher test (node size=20) using all genes, each marked for presence or absence in the set of putative immune genes. We then tested for enrichment of the most differentially expressed genes between sexes and pesticide treatment groups. For this approach, we ranked genes by unadjusted raw $p$ values to avoid edge-effects introduced by correction and performed a rank-based analysis (Kolmogorov-Smirnov test with "weighted01" algorithm and a node size=20). We also performed an immune-focused GO term enrichment analysis where we populated our GO term database only with genes annotated with immune-related GO terms.

\section{Data and Code Availability}

Data used for the present analysis originated from datasets generated by (Beadle et al., 2019). 


\section{References}

Adams, M. D. (2000) 'The genome sequence of Drosophila melanogaster', Science, pp. 21852195. doi: 10.1126/science.287.5461.2185.

Albert, Š. et al. (2011) 'Evidence of a novel immune responsive protein in the Hymenoptera', Insect biochemistry and molecular biology, 41(12), pp. 968-981.

Alexa, A. and Rahnenführer, J. (2009) 'Gene set enrichment analysis with topGO', Bioconductor Improv, 27. Available at: http://bioconductor.riken.jp/packages/3.0/bioc/vignettes/topGO/inst/doc/ topGO.pdf.

Andrews, S. (2010) 'FastQC: a quality control tool for high throughput sequence data', Reference Source. Available at: http://www.bioinformatics.babraham.ac.uk/projects/fastqc/.

Arai, I. et al. (2013) 'Immunohistochemical analysis of the role of hemocytin in nodule formation in the larvae of the silkworm, Bombyx mori', Journal of insect science , 13, p. 125.

Arce, A. N. et al. (2017) 'Impact of controlled neonicotinoid exposure on bumblebees in a realistic field setting', The Journal of applied ecology. Edited by M. Pocock, 54(4), pp. 1199-1208.

Arce, A. N. et al. (2018) 'Foraging bumblebees acquire a preference for neonicotinoid-treated food with prolonged exposure', Proceedings. Biological sciences / The Royal Society, 285(1885). doi: 10.1098/rspb.2018.0655.

Azpiazu, C. et al. (2019) 'Chronic oral exposure to field-realistic pesticide combinations via pollen and nectar: effects on feeding and thermal performance in a solitary bee', Scientific reports, 9(1), p. 13770.

Baer, B. et al. (2005) 'Examination of the immune responses of males and workers of the leafcutting ant Acromyrmex echinatior and the effect of infection', Insectes sociaux, 52(3), pp. 298303.

Barribeau, S. M. et al. (2015) 'A depauperate immune repertoire precedes evolution of sociality in bees', Genome biology, 16, p. 83.

Barribeau, S. M. and Schmid-Hempel, P. (2017) 'Sexual healing: mating induces a protective immune response in bumblebees', Journal of evolutionary biology, 30(1), pp. 202-209.

Beadle, K. et al. (2019) 'Genomic insights into neonicotinoid sensitivity in the solitary bee Osmia bicornis', PLoS genetics, 15(2), p. e1007903.

Bebane, P. S. A. et al. (2019) 'The effects of the neonicotinoid imidacloprid on gene expression and DNA methylation in the buff-tailed bumblebee Bombus terrestris', Proceedings. Biological sciences / The Royal Society, 286(1905), p. 20190718.

Beckage, N. E. (2008) 'Insect immunology'. Academic Press, New York.

Blacquière, T. et al. (2012) 'Neonicotinoids in bees: a review on concentrations, side-effects and risk assessment', Ecotoxicology , 21(4), pp. 973-992.

Boff, S. et al. (2021) 'Survival rate and changes in foraging performances of solitary bees exposed to a novel insecticide', Ecotoxicology and environmental safety, 211, p. 111869.

Bonasio, R. et al. (2010) 'Genomic comparison of the ants Camponotus floridanus and 
Harpegnathos saltator', Science, 329(5995), pp. 1068-1071.

Bramke, K. et al. (2019) 'Exposure of larvae of the solitary bee Osmia bicornis to the honey bee pathogen Nosema ceranae affects life history', Insects, 10(11). doi: 10.3390/insects10110380.

Brandt, A. et al. (2017) 'Immunosuppression in honeybee queens by the neonicotinoids thiacloprid and clothianidin', Scientific reports, 7(1), p. 4673.

Brandt, A. et al. (2020) 'Immunosuppression response to the neonicotinoid insecticide thiacloprid in females and males of the red mason bee Osmia bicornis L', Scientific reports, 10(1), p. 4670.

Bray, N. L. et al. (2016) 'Near-optimal probabilistic RNA-seq quantification', Nature biotechnology, 34(5), pp. 525-527.

Brown, M. J. F. and Paxton, R. J. (2009) 'The conservation of bees: a global perspective', Apidologie, 40(3), pp. 410-416.

Brutscher, L. M., Daughenbaugh, K. F. and Flenniken, M. L. (2015) 'Antiviral defense mechanisms in honey bees', Current opinion in insect science, 10, pp. 71-82.

Calderone, N. W. (2012) 'Insect pollinated crops, insect pollinators and US agriculture: Trend analysis of aggregate data for the period 1992-2009', PLOS ONE, p. e37235. doi: 10.1371/journal.pone.0037235.

Calleri, D. V. et al. (2006) 'Inbreeding and disease resistance in a social insect: effects of heterozygosity on immunocompetence in the termite Zootermopsis angusticollis', Proceedings of the Royal Society B: Biological Sciences, pp. 2633-2640. doi: 10.1098/rspb.2006.3622.

Casteels, P. et al. (1990) 'Isolation and characterization of abaecin, a major antibacterial response peptide in the honeybee (Apis mellifera)', European Journal of Biochemistry, pp. 381-386. doi: 10.1111/j.1432-1033.1990.tb15315.x.

Casteels, P. et al. (1993) 'Functional and chemical characterization of Hymenoptaecin, an antibacterial polypeptide that is infection-inducible in the honeybee (Apis mellifera)', The Journal of biological chemistry, 268(10), pp. 7044-7054.

Chaimanee, V. et al. (2016) 'Sperm viability and gene expression in honey bee queens (Apis mellifera) following exposure to the neonicotinoid insecticide imidacloprid and the organophosphate acaricide coumaphos', Journal of insect physiology, 89, pp. 1-8.

Chen, J., Nolte, V. and Schlötterer, C. (2015) 'Temperature-related reaction norms of gene expression: Regulatory architecture and functional implications', Molecular biology and evolution, 32(9), pp. 2393-2402.

Chen, S. et al. (2018) 'fastp: an ultra-fast all-in-one FASTQ preprocessor', Bioinformatics , 34(17), pp. i884-i890.

Chmiel, J. A. et al. (2019) 'Deleterious effects of neonicotinoid pesticides on Drosophila melanogaster immune pathways', mBio, 10(5). doi: 10.1128/mBio.01395-19.

Christophides, G. K. et al. (2002) 'Immunity-related genes and gene families in Anopheles gambiae', Science, 298(5591), pp. 159-165.

Claus, G. et al. (2021) 'Larval oral exposure to thiacloprid: Dose-response toxicity testing in solitary bees, Osmia spp. (Hymenoptera: Megachilidae)', Ecotoxicology and environmental safety, 215, p. 112143. 
Colgan, T. J. et al. (2011) 'Polyphenism in social insects: insights from a transcriptome-wide analysis of gene expression in the life stages of the key pollinator, Bombus terrestris', BMC genomics, 12, p. 623.

Colgan, T. J. et al. (2019) 'Mating induces selective immune priming which is maintained throughout bumblebee queen diapause', BMC Genomics. Available at: https://europepmc.org/article/ppr/ppr143125.

Colinet, D. et al. (2011) 'Extracellular superoxide dismutase in insects: characterization, function, and interspecific variation in parasitoid wasp venom', The Journal of biological chemistry, 286(46), pp. 40110-40121.

Combes, C. (2001) Parasitism: The ecology and evolution of intimate interactions. University of Chicago Press.

DeGrandi-Hoffman, G. and Chen, Y. (2015) 'Nutrition, immunity and viral infections in honey bees', Current opinion in insect science, 10, pp. 170-176.

De Gregorio, E. et al. (2002) 'The Toll and Imd pathways are the major regulators of the immune response in Drosophila', The EMBO journal, 21(11), pp. 2568-2579.

Di Prisco, G. et al. (2013) 'Neonicotinoid clothianidin adversely affects insect immunity and promotes replication of a viral pathogen in honey bees', Proceedings of the National Academy of Sciences of the United States of America, 110(46), pp. 18466-18471.

Dmochowska-Ślęzak, K. et al. (2015) 'Variations in antioxidant defense during the development of the solitary bee Osmia bicornis', Apidologie, 46(4), pp. 432-444.

Dobin, A. et al. (2013) 'STAR: ultrafast universal RNA-seq aligner', Bioinformatics , 29(1), pp. 1521.

Dong, J. et al. (2020) 'Novel characteristics of immune responsive protein IRP30 in the bumble bee Bombus lantschouensis (Hymenoptera: Apidae)', Journal of insect science , 20(2). doi: 10.1093/jisesa/ieaa017.

Durinck, S. et al. (2009) 'Mapping identifiers for the integration of genomic datasets with the R/Bioconductor package biomaRt', Nature protocols, 4(8), pp. 1184-1191.

Emms, D. M. and Kelly, S. (2019) 'OrthoFinder: phylogenetic orthology inference for comparative genomics', Genome biology, 20(1), p. 238.

European Red List of Bees (2014).

Evans, J. D. et al. (2006) 'Immune pathways and defence mechanisms in honey bees Apis mellifera', Insect molecular biology, 15(5), pp. 645-656.

Ewels, P. et al. (2016) 'MultiQC: summarize analysis results for multiple tools and samples in a single report', Bioinformatics , 32(19), pp. 3047-3048.

Fish, E. N. (2008) 'The X-files in immunity: sex-based differences predispose immune responses', Nature reviews. Immunology, 8(9), pp. 737-744.

Galiana-Arnoux, D. et al. (2006) 'Essential function in vivo for Dicer-2 in host defense against RNA viruses in Drosophila', Nature immunology, 7(6), pp. 590-597.

Gallai, N. et al. (2009) 'Economic valuation of the vulnerability of world agriculture confronted with pollinator decline', Ecological economics: the journal of the International Society for Ecological 
Economics, 68(3), pp. 810-821.

Gerardo, N. M. et al. (2010) 'Immunity and other defenses in pea aphids, Acyrthosiphon pisum', Genome biology, 11(2), p. R21.

Gill, R. J., Ramos-Rodriguez, O. and Raine, N. E. (2012) 'Combined pesticide exposure severely affects individual- and colony-level traits in bees', Nature, 491(7422), pp. 105-108.

Goulson, D. et al. (2015) 'Bee declines driven by combined stress from parasites, pesticides, and lack of flowers', Science, 347(6229), p. 1255957.

Gruber, B. et al. (2011) 'On managing the red mason bee (Osmia bicornis) in apple orchards', Apidologie, 42(5). doi: 10.1007/s13592-011-0059-z.

Harpur, B. A. and Zayed, A. (2013) 'Accelerated evolution of innate immunity proteins in social insects: Adaptive evolution or relaxed constraint?', Molecular Biology and Evolution, pp. 16651674. doi: $10.1093 / \mathrm{molbev} / \mathrm{mst} 061$.

Hill-Burns, E. M. and Clark, A. G. (2009) 'X-linked variation in immune response in Drosophila melanogaster', Genetics, 183(4), pp. 1477-1491.

Hoffmann, J. A. (1995) 'Innate immunity of insects', Current Opinion in Immunology, pp. 4-10. doi: 10.1016/0952-7915(95)80022-0.

Hoffmann, J. A. and Hetru, C. (1992) 'Insect defensins: inducible antibacterial peptides', Immunology Today, pp. 411-415. doi: 10.1016/0167-5699(92)90092-I.

Imler, J.-L. and Bulet, P. (2005) 'Antimicrobial peptides in Drosophila: structures, activities and gene regulation', Chemical immunology and allergy, 86, pp. 1-21.

Ingersoll, M. A. (2017) 'Sex differences shape the response to infectious diseases', PLoS pathogens, 13(12), p. e1006688.

James, R. R. and Xu, J. (2012) 'Mechanisms by which pesticides affect insect immunity', Journal of invertebrate pathology, 109(2), pp. 175-182.

Jeschke, P. and Nauen, R. (2008) 'Neonicotinoids-from zero to hero in insecticide chemistry', Pest management science, 64(11), pp. 1084-1098.

Jiggins, F. M. and Kim, K. W. (2007) 'A screen for immunity genes evolving under positive selection in Drosophila', Journal of evolutionary biology, 20(3), pp. 965-970.

Jones, P. et al. (2014) 'InterProScan 5: genome-scale protein function classification', Bioinformatics , 30(9), pp. 1236-1240.

Joseph, S. B. and Kirkpatrick, M. (2004) 'Haploid selection in animals', Trends in ecology \& evolution, 19(11), pp. 592-597.

Kinsella, R. J. et al. (2011) 'Ensembl BioMarts: a hub for data retrieval across taxonomic space', Database: the journal of biological databases and curation, 2011, p. bar030.

Klein, A.-M., Steffan-Dewenter, I. and Tscharntke, T. (2003) 'Fruit set of highland coffee increases with the diversity of pollinating bees', Proceedings. Biological sciences / The Royal Society, 270(1518), pp. 955-961.

Klein, S. L. and Flanagan, K. L. (2016) 'Sex differences in immune responses', Nature reviews. Immunology, 16(10), pp. 626-638. 
Korayem, A. M. et al. (2004) 'A Drosophila salivary gland mucin is also expressed in immune tissues: evidence for a function in coagulation and the entrapment of bacteria', Insect biochemistry and molecular biology, 34(12), pp. 1297-1304.

Kubrak, O. I. et al. (2014) 'The sleeping beauty: how reproductive diapause affects hormone signaling, metabolism, immune response and somatic maintenance in Drosophila melanogaster', PloS one, 9(11), p. e113051.

Lavine, M. D. and Strand, M. R. (2002) 'Insect hemocytes and their role in immunity', Insect Biochemistry and Molecular Biology, pp. 1295-1309. doi: 10.1016/s0965-1748(02)00092-9.

Lawniczak, M. K. N. et al. (2007) 'Mating and immunity in invertebrates', Trends in ecology \& evolution, 22(1), pp. 48-55.

Losey, J. E. and Vaughan, M. (2006) 'The economic value of ecological services provided by insects', Bioscience, 56(4), pp. 311-323.

Love, M. I., Huber, W. and Anders, S. (2014) 'Moderated estimation of fold change and dispersion for RNA-seq data with DESeq2', Genome biology, 15(12), p. 550.

Manjon, C. et al. (2018) 'Unravelling the molecular determinants of bee sensitivity to neonicotinoid insecticides', Current biology: CB, 28(7), pp. 1137-1143.e5.

Mason, R. et al. (2013) 'Immune suppression by neonicotinoid insecticides at the root of global wildlife declines', Journal of environmental immunology and toxicology, 1(1), p. 3.

Matsuda, K. et al. (2001) 'Neonicotinoids: insecticides acting on insect nicotinic acetylcholine receptors', Trends in pharmacological sciences, 22(11), pp. 573-580.

Moffat, C. et al. (2015) 'Chronic exposure to neonicotinoids increases neuronal vulnerability to mitochondrial dysfunction in the bumblebee (Bombus terrestris)', FASEB journal: official publication of the Federation of American Societies for Experimental Biology, 29(5), pp. 2112-2119.

Moffat, C. et al. (2016) 'Neonicotinoids target distinct nicotinic acetylcholine receptors and neurons, leading to differential risks to bumblebees', Scientific reports, 6, p. 24764.

Mokkapati, J. S., Bednarska, A. J. and Laskowski, R. (2021) 'The development of the solitary bee Osmia bicornis is affected by some insecticide agrochemicals at environmentally relevant concentrations', The Science of the total environment, 775, p. 145588.

Moret, Y. and Schmid-Hempel, P. (2000) 'Survival for immunity: the price of immune system activation for bumblebee workers', Science, 290(5494), pp. 1166-1168.

Nakamura, A. et al. (2011) 'Innate immune system still works at diapause, a physiological state of dormancy in insects', Biochemical and biophysical research communications, 410(2), pp. 351-357.

'Neonicotinoids, bee disorders and the sustainability of pollinator services' (2013) Current Opinion in Environmental Sustainability, 5(3-4), pp. 293-305.

Ni, W. et al. (2020) 'Hemocytin facilitates host immune responses against Nosema bombycis', Developmental and comparative immunology, 103, p. 103495.

O'Donnell, S. and Beshers, S. N. (2004) 'The role of male disease susceptibility in the evolution of haplodiploid insect societies', Proceedings. Biological sciences / The Royal Society, 271(1542), pp. 979-983.

Pace, K. E. and Baum, L. G. (2002) 'Insect galectins: roles in immunity and development', 
Glycoconjugate journal, 19(7-9), pp. 607-614.

Pamilo, P. and Crozier, R. H. (1981) 'Genic variation in male haploids under deterministic selection', Genetics, 98(1), pp. 199-214.

Parsch, J. and Ellegren, H. (2013) 'The evolutionary causes and consequences of sex-biased gene expression', Nature reviews. Genetics, 14(2), pp. 83-87.

Peng, J., Zipperlen, P. and Kubli, E. (2005) 'Drosophila sex-peptide stimulates female innate immune system after mating via the Toll and Imd pathways', Current biology: CB, 15(18), pp. 1690-1694.

Rao, X.-J. et al. (2016) 'Characterization of a dual-CRD galectin in the silkworm Bombyx mori', Developmental and comparative immunology, 60, pp. 149-159.

Rees, J. A., Moniatte, M. and Bulet, P. (1997) 'Novel antibacterial peptides isolated from a European bumblebee, Bombus pascuorum (Hymenoptera, Apoidea)', Insect biochemistry and molecular biology, 27(5), pp. 413-422.

Retschnig, G. et al. (2014) 'Sex-specific differences in pathogen susceptibility in honey bees (Apis mellifera)', PloS one, 9(1), p. e85261.

Rogers, L. J., Frasnelli, E. and Versace, E. (2016) 'Lateralized antennal control of aggression and sex differences in red mason bees, Osmia bicornis', Scientific reports, 6, p. 29411.

Rolff, J. and Reynolds, S. (2009) Insect infection and immunity: Evolution, ecology, and mechanisms. OUP Oxford.

Rolff, J. and Siva-Jothy, M. T. (2003) 'Invertebrate ecological immunology', Science, 301(5632), pp. 472-475.

Roux, J. et al. (2014) 'Patterns of positive selection in seven ant genomes', Molecular biology and evolution, 31(7), pp. 1661-1685.

Ruddle, N. et al. (2018) 'Effects of exposure to winter oilseed rape grown from thiamethoxamtreated seed on the red mason bee Osmia bicornis', Environmental toxicology and chemistry / SETAC, 37(4), pp. 1071-1083.

Ruiz-González, M. X. and Brown, M. J. F. (2006) 'Males vs workers: testing the assumptions of the haploid susceptibility hypothesis in bumblebees', Behavioral ecology and sociobiology, 60(4), pp. 501-509.

Sackton, T. B. et al. (2007) 'Dynamic evolution of the innate immune system in Drosophila', Nature genetics, 39(12), pp. 1461-1468.

Sadd, B. M. et al. (2015) 'The genomes of two key bumblebee species with primitive eusocial organization', Genome biology, 16, p. 76.

Sadd, B. M. and Schmid-Hempel, P. (2008) 'PERSPECTIVE: Principles of ecological immunology', Evolutionary applications, 2(1), pp. 113-121.

Sadd, B. M. and Schmid-Hempel, P. (2009) 'Ecological and evolutionary implications of specific immune responses', Insect Infection and Immunity, pp. 225-240. doi:

10.1093/acprof:oso/9780199551354.003.0014.

Sandrock, C. et al. (2014) 'Sublethal neonicotinoid insecticide exposure reduces solitary bee reproductive success', Agricultural and forest entomology, 16(2), pp. 119-128. 
Schoonvaere, K. et al. (2018) 'Study of the metatranscriptome of eight social and solitary wild bee species reveals novel viruses and bee parasites', Frontiers in microbiology, 9, p. 177.

Schwenke, R. A., Lazzaro, B. P. and Wolfner, M. F. (2016) 'Reproduction-immunity trade-offs in insects', Annual review of entomology, 61, pp. 239-256.

Seidelmann, K. (2006) 'Open-cell parasitism shapes maternal investment patterns in the red mason bee Osmia rufa', Behavioral Ecology, pp. 839-848. doi: 10.1093/beheco/arl017.

Shen, S. et al. (2014) 'rMATS: robust and flexible detection of differential alternative splicing from replicate RNA-Seq data', Proceedings of the National Academy of Sciences of the United States of America, 111(51), pp. E5593-601.

Shultz, A. J. and Sackton, T. B. (2019) 'Immune genes are hotspots of shared positive selection across birds and mammals', eLife, 8. doi: 10.7554/eLife.41815.

Shumate, A. and Salzberg, S. L. (2020) 'Liftoff: accurate mapping of gene annotations', Bioinformatics . doi: 10.1093/bioinformatics/btaa1016.

Siviter, H. et al. (2018) 'Quantifying the impact of pesticides on learning and memory in bees', The Journal of applied ecology. Edited by M. Pocock, 19, p. 915.

Stanley, D. A., Smith, K. E. and Raine, N. E. (2015) 'Bumblebee learning and memory is impaired by chronic exposure to a neonicotinoid pesticide', Scientific reports, 5, p. 16508.

Straub, F. et al. (2021) 'Negative effects of the neonicotinoid clothianidin on foraging behavior and antennal sensitivity in two common pollinator species, Osmia bicornis and Bombus terrestris', Frontiers in Ecology and Evolution, 9. doi: 10.3389/fevo.2021.697355.

Szentgyörgyi, H. et al. (2017) 'Forewing structure of the solitary bee Osmia bicornis developing on heavy metal pollution gradient', Ecotoxicology , 26(8), pp. 1031-1040.

Tian et al. (2018) 'Infection with the multi-host micro-parasite Apicystis bombi (Apicomplexa: Neogregarinorida) decreases survival of the solitary bee Osmia bicornis', Journal of Invertebrate Pathology, pp. 43-45. doi: 10.1016/j.jip.2018.09.005.

Troczka, B. J. et al. (2019) 'Identification and functional characterisation of a novel N-cyanoamidine neonicotinoid metabolising cytochrome P450, CYP9Q6, from the buff-tailed bumblebee Bombus terrestris', Insect biochemistry and molecular biology, 111, p. 103171.

Vanengelsdorp, D. and Meixner, M. D. (2010) 'A historical review of managed honey bee populations in Europe and the United States and the factors that may affect them', Journal of invertebrate pathology, 103 Suppl 1, pp. S80-95.

Viljakainen, L. et al. (2009) 'Rapid evolution of immune proteins in social insects', Molecular biology and evolution, 26(8), pp. 1791-1801.

Viljakainen, L. (2015) 'Evolutionary genetics of insect innate immunity', Briefings in Functional Genomics, pp. 407-412. doi: 10.1093/bfgp/elv002.

Waterhouse, R. M. et al. (2007) 'Evolutionary dynamics of immune-related genes and pathways in disease-vector mosquitoes', Science, 316(5832), pp. 1738-1743.

Werren, J. H. et al. (2010) 'Functional and evolutionary insights from the genomes of three parasitoid Nasonia species', Science, 327(5963), pp. 343-348.

Woodcock, B. A. et al. (2017) 'Country-specific effects of neonicotinoid pesticides on honey bees 
bioRxiv preprint doi: https://doi.org/10.1101/2021.10.04.462642; this version posted October 5, 2021. The copyright holder for this preprint (which was not certified by peer review) is the author/funder, who has granted bioRxiv a license to display the preprint in perpetuity. It is made available under aCC-BY-NC-ND 4.0 International license.

827 and wild bees', Science, 356(6345), pp. 1393-1395.

$828 \mathrm{Xu}, \mathrm{J}$. and James, R. R. (2012) 'Temperature stress affects the expression of immune response genes in the alfalfa leafcutting bee, Megachile rotundata', Insect molecular biology, 21(2), pp. 269280. 


\section{Figures}

A
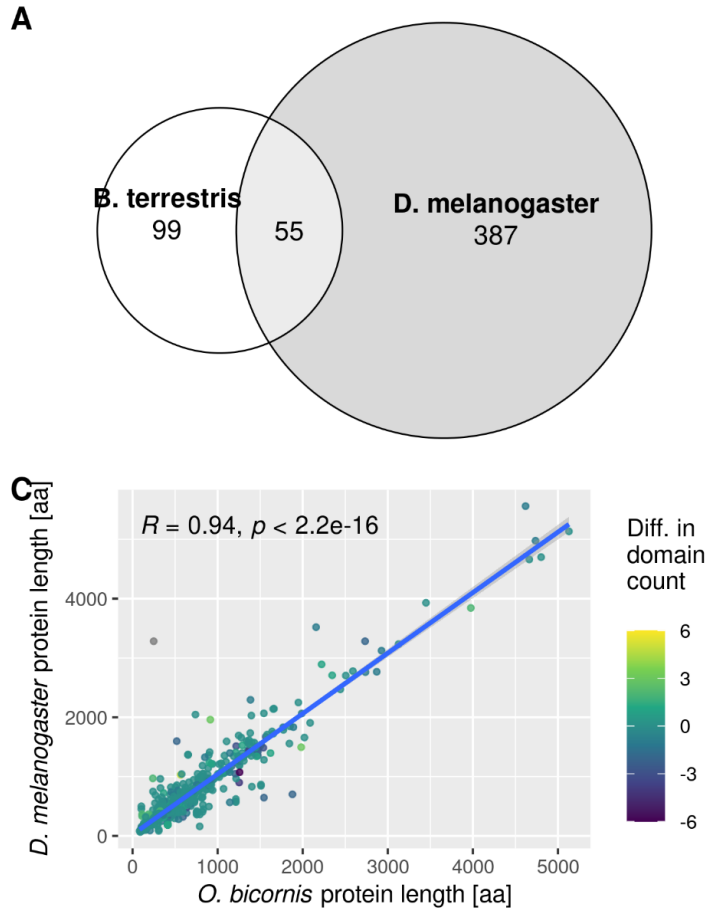

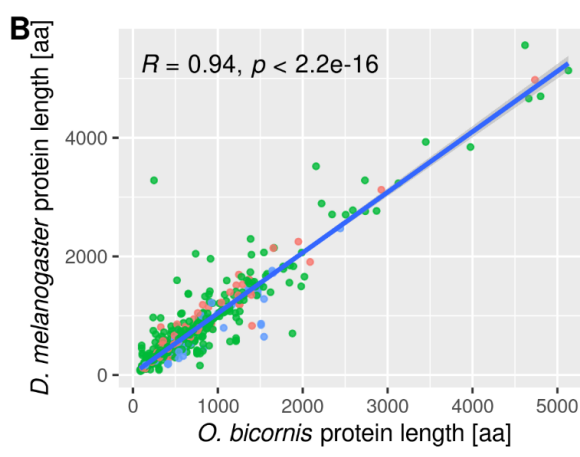

D

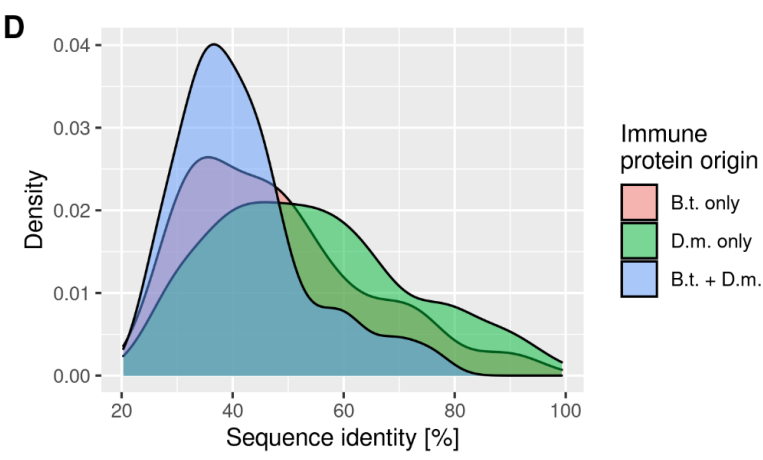

Figure 1. Expanded repertoire of putative immune genes in the red mason bee, Osmia bicornis. (A) Overlap of putative $O$. bicornis immune genes inferred via homology to $B$. terrestris and $D$. melanogaster. (BC) Correlation of protein lengths (amino acids) and number of functional domains shared between $O$. bicornis and $D$. melanogaster protein homologues with colours indicating (B) immune gene set where homology was found ("B.t. only" = homologues identified through comparison with B. terrestris; "D.m. only" = homologues identified through comparison with D. melanogaster; "B.t.+D.m." = homologues identified in comparisons with both species) and (C) difference in number of domains (yellow: higher domain count in $D$. melanogaster, purple: higher domain count in O. bicornis). (D) Distribution of sequence identity (percentage of identical amino acid positions) of homologous protein pairs between $D$. melanogaster and O. bicornis for putative $\mathrm{O}$. bicornis immune proteins. Colours indicate the species through which homologues were identified. 


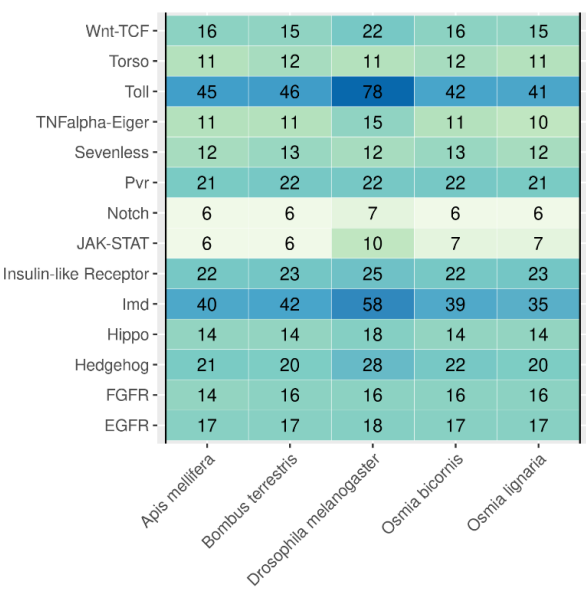

B

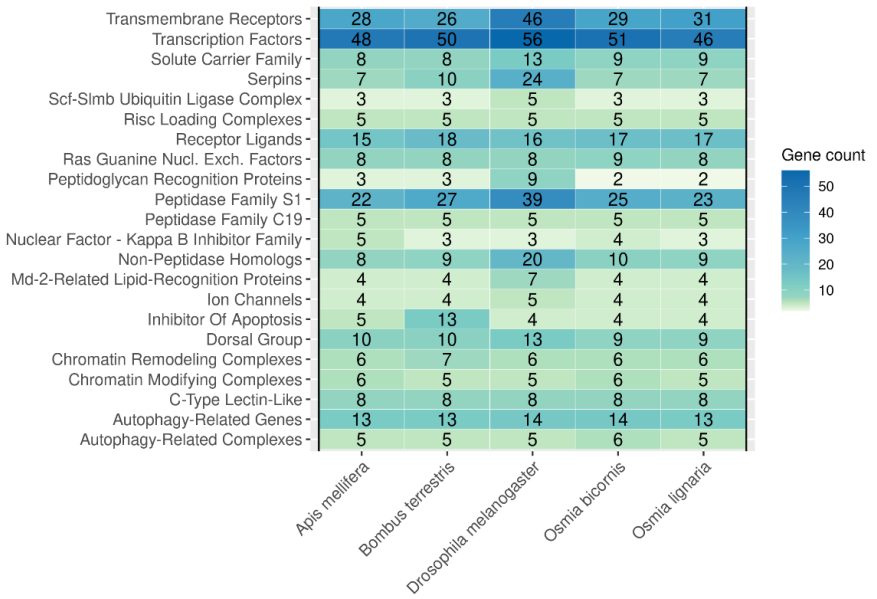

Figure 2. Conservation of immune genes and pathways in Osmia bicornis. Heatmaps depicting gene counts of homologues of putative $\mathrm{O}$. bicornis immune genes for (A) molecular signaling pathways and (B) non-pathway gene families in closely related hymenopterans (Apis mellifera, Bombus terrestris, Osmia lignaria) and the more distantly related Drosophila melanogaster. For each species the number of homologues are shown. 
A

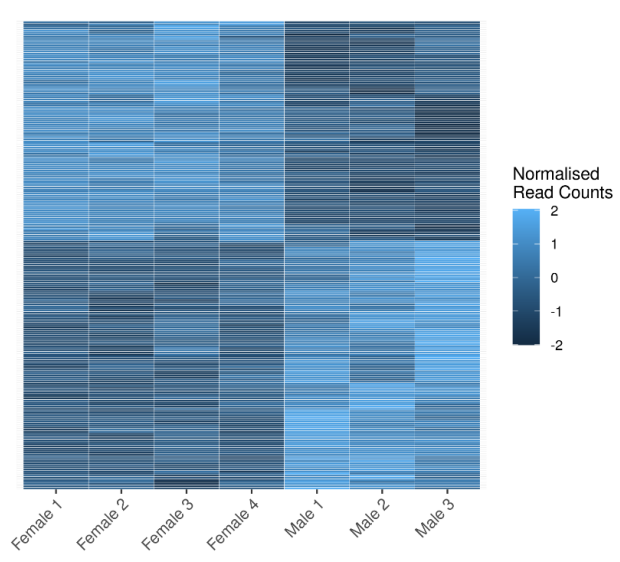

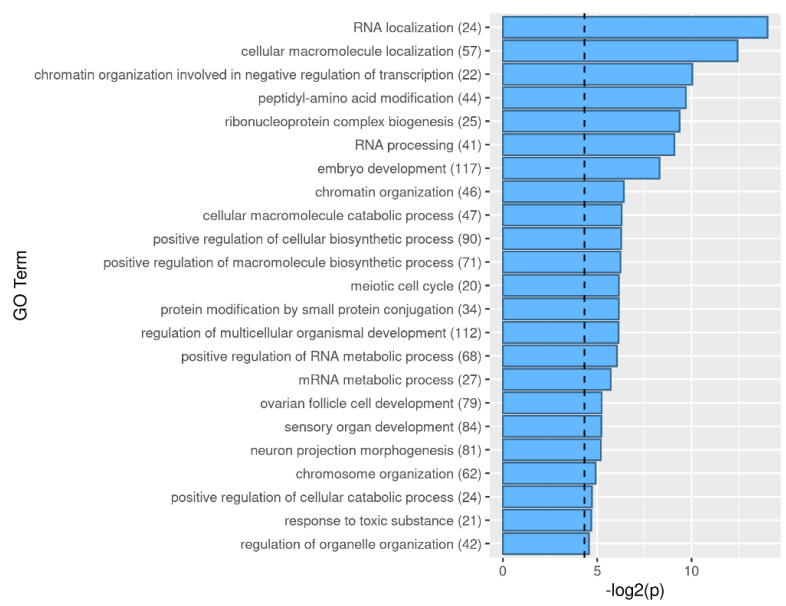

Figure 3. Differential expression of putative Osmia bicornis immune genes between males and females. (A) Heatmap showing normalised (variance stabilisation transformed) read counts per sample for significantly differentially expressed (BH adjusted $p<0.05)$ immune genes between males and females. (B) Functional enrichment of biological process $G O$ terms of significantly differentially expressed putative $O$. bicornis immune genes. The $\mathrm{x}$ axis depicts negative log-transformed $p$ values with the dashed vertical line corresponding to a $p$ value confidence threshold of 0.05 . Each line on the $y$ axis corresponds to a GO term description with the numbers in brackets indicating the number of genes annotated with that specific term in the GO term database. 
A

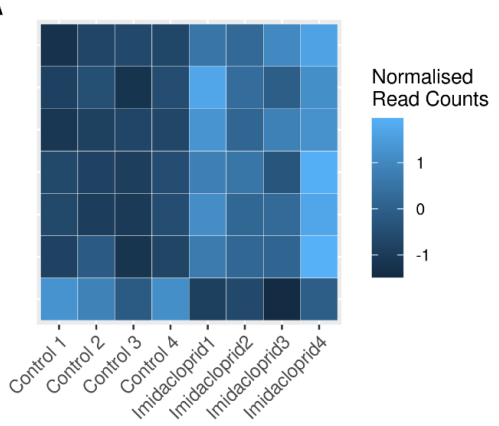

C

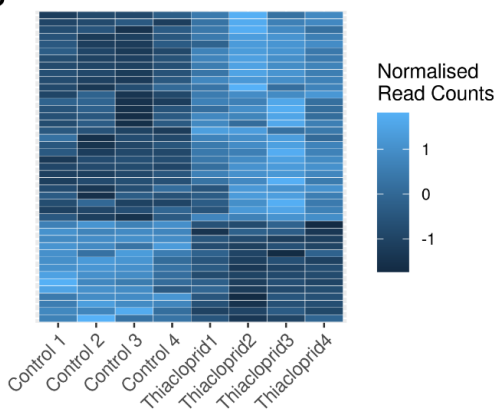

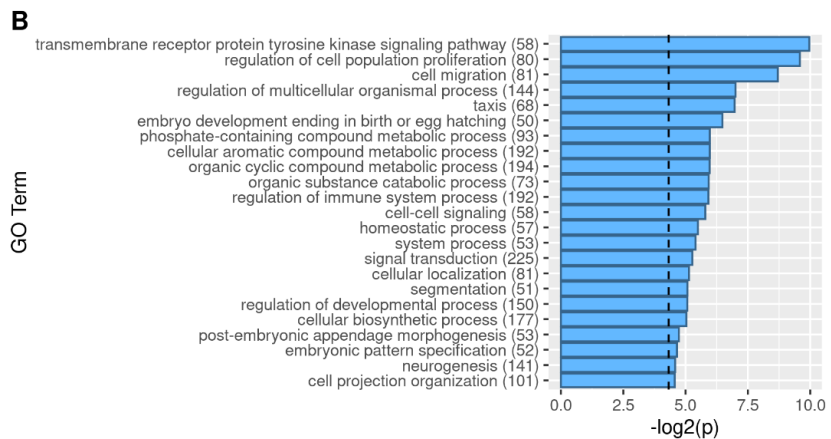

D

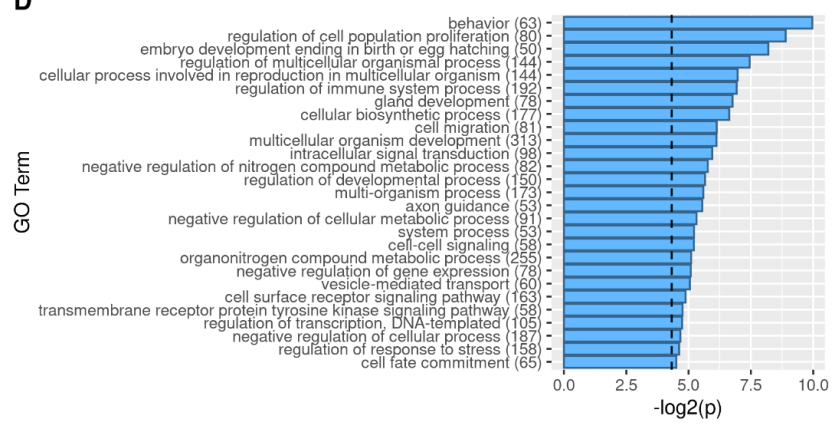

Figure 4. Differential expression of putative Osmia bicornis immune genes between pesticideexposed and control individuals. (A,C) Heatmap showing normalised (variance stabilisation transformed) read counts per sample for significantly differentially expressed ( $\mathrm{BH}$ adjusted $p<0.05$ ) immune genes between control individuals and (A) imidacloprid-treated or (C) thiacloprid-treated individuals. (B,D) Functional enrichment of biological process GO terms of significantly differentially expressed putative $O$. bicornis immune genes between control individuals and (B) imidacloprid-treated individuals or (D) thiaclopridtreated individuals. The $\mathrm{x}$ axis depicts negative log-transformed $p$ values with the dashed vertical line corresponding to a $p$ value confidence threshold of 0.05 . Each line on the $y$ axis corresponds to a GO term description with the numbers in brackets indicating the number of genes annotated with that specific term in the GO term database. 